\title{
A New N2N Packet Forwarding Technique for MPEG Video Transport in IP networks
}

\author{
V. B. Dharmadhikari \\ Associate Professor \\ Department of Electronics Engineering \\ Walchand College of Engineering, Sangli, India
}

\author{
P. J. Kulkarni \\ Professor \\ Department of Computer Science and Engineering \\ Walchand College of Engineering, Sangli, India
}

\begin{abstract}
Due to increase in the multimedia applications and streaming servers, transport of MPEG compressed video data through a packet switched network has become highly important. It is necessary to transport the video while ensuring appropriate video quality at the receiving end. This paper proposes a new $\mathrm{N} 2 \mathrm{~N}$ packet forwarding technique that reduces the packet loss and delay jitter of the video traffic. The paper also shows that with the use of the proposed packet forwarding technique, the PSNR is also improved.
\end{abstract}

\section{General Terms}

Video Transport, Multimedia Communication

\section{Keywords}

MPEG, Video, Packet forwarding, Quality of Service

\section{INTRODUCTION}

In recent years, multimedia applications are seen increase in their usage. These include live video telecasts, webinars, YouTube like video streaming services, news streaming and so on. These applications require certain quality of service. This can be ensured with low packet loss and low delay variance (jitter). If the packet loss and jitter are low, the playing of video at the receiving end is smoother and hence the user experience will be better.

The video transport in any packet switched network consists of first converting the analog light intensities at each point in a picture into digital form. Then, the digital information is compressed using compression techniques. A well accepted video compression standard is the MPEG compression standard [1]. The compression is applied on each video frame. Due to the nature of MPEG compression, the amount of data corresponding to each frame may change depending on the video content of that frame and its co-relation with the nearby frames [2]. This creates VBR traffic for an MPEG compressed video.

For sending the video data through a packet switched network such as Internet, the data of each frame needs to be packetized. The packets are then sent to the receiving end through various packet forwarding nodes in the Internet. Along with video packets, these nodes also carry other nonvideo data packets such as ftp, http traffic etc. In this paper, the non-video traffic is referred as data traffic. Each packet forwarding node in Internet processes the incoming packet and sends it to the appropriate next node. While doing this it may use same packet forwarding technique to all packets or it may use different techniques for different types of packets. For example, it may give higher priority to video packets so as to reduce the delay between the sender and receiver. Also it is necessary to reduce the jitter; otherwise it may cause impairments in the received video [3]. The packet loss for video traffic can cause degradation in the quality of received video [4]. Therefore it is necessary to device a packet forwarding mechanism that will reduce packet loss as well as delay jitter.

The Internet being a best effort packet forwarding network, is unsuitable to guarantee certain packet loss and packet delay. However, Internet being widespread, it is easier and economical to use it for video services also. In this context, the packet forwarding techniques are vital since they decide the quality of service parameters such as packet loss, end-toend packet delay etc.

The paper is arranged as follows: In section 2, we review the present packet forwarding techniques. Section 3 discusses the proposed N2N technique, Section 4 presents the results and section 5 presents the conclusion and future scope.

\section{PREVIOUS WORK}

In Internet, the network layer uses Internet Protocol (IP). This protocol does the best effort packet forwarding. If a packet is dropped due to congestion, then the IP does not try to recover the loss, but end application has to take care of such packet losses. To avoid such packet loss, the applications use the Transport Control Protocol (TCP). This protocol is an end to end protocol which relies on retransmission of packets which are dropped in the network. Due to retransmission of dropped packets at the TCP layer, the effective packet loss for the application that uses TCP is zero.

There have been a few of packet forwarding techniques suggested for video data. Biswaroop Mukherjee [5] has proposed an end to end protocol which is a variant of TCP, and is called as Time-lined TCP (TL-TCP). His work uses TCP, with a time out period for re-transmission. Original TCP continues to request for retransmission till all the data is correctly received without any time limit. This may result in correct packets received but these may be so late that the video data in these late packets cannot be played and thus it becomes useless video data. The TL-TCP solves this problem by introducing a time out period. However, it is still an end to end protocol. Hence it cannot reduce the delay of the retransmitted packets if the round trip time (RTT) delay is large. Our proposed $\mathrm{N} 2 \mathrm{~N}$ technique reduces the delay of retransmitted packets, even under large RTT, further reducing the jitter also. 


\section{PROPOSED WORK}

\subsection{System Model}

We consider an IP network (figure 1) with $\mathrm{K}$ nodes between the video sender (source) and receiver (sink). Each node uses a FIFO type of buffering. The model of a node is shown in figure 2. The node model consists of a main buffer (MB), and a retransmission buffer (RB). The main buffer is used to enque the arrived packets. When a packet at the head of the buffer is serviced, then if it is a video data packet, then its copy is put into the retransmission buffer for future use.

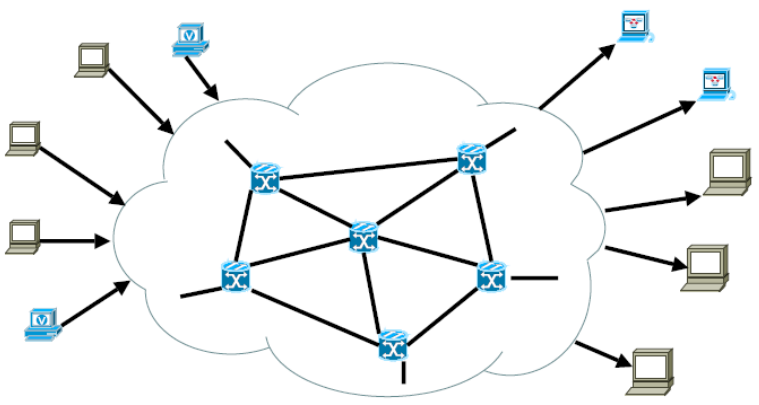

We assume that, the video packets contain a flow id, by which it is possible for each node to identify that a packet is a video packet. Also we assume that, the video packet contains a flow sequence number.

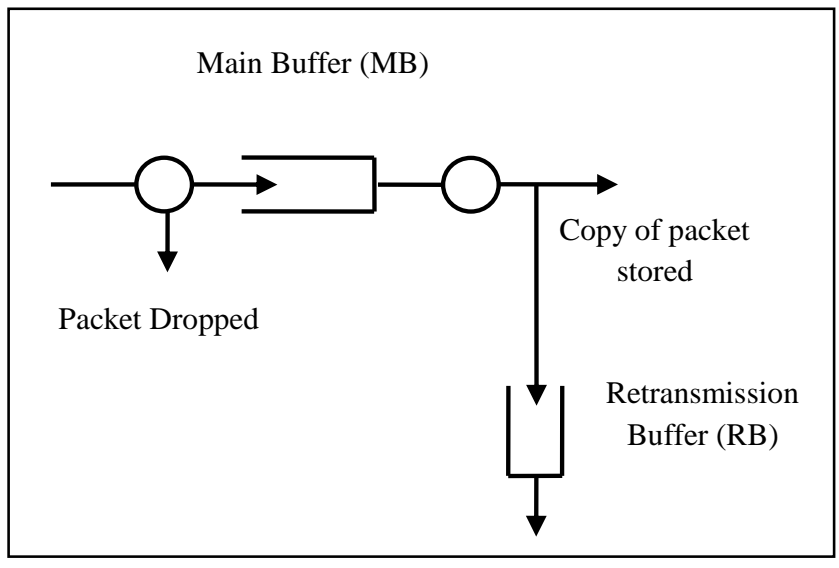

Figure 2: Node Model

Figure 1: IP network for packet forwarding

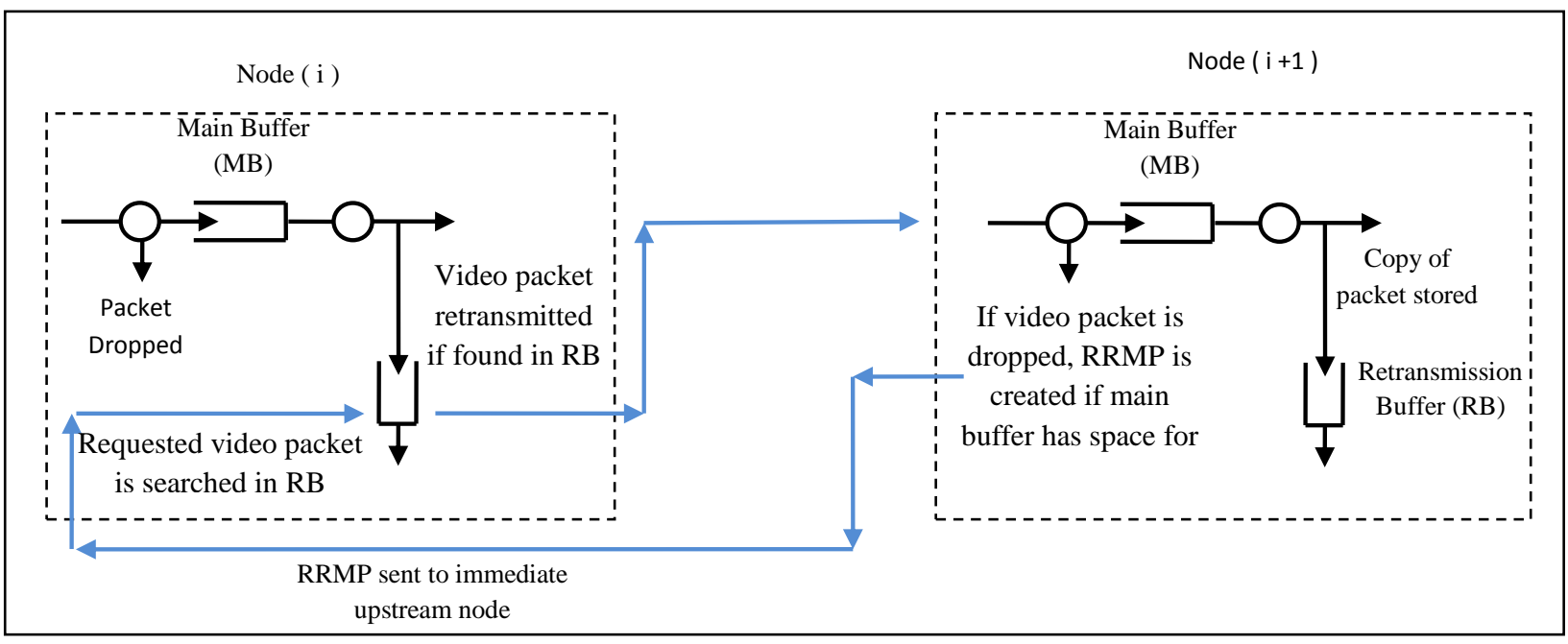

\section{Figure 3: Node to Node (N2N) re-transmission algorithm}

\subsection{N2N Algorithm}

The operation of the propose $\mathrm{N} 2 \mathrm{~N}$ packet forwarding is as follows:

The proposed N2N re-transmission based packet forwarding algorithm uses node to node retransmission unlike the TCP or TL-TCP which use end-to-end retransmission. For node to node retransmission, each node uses a retransmission buffer (RB) to keep copy of the transmitted video packet. The retransmission buffer is used only for video packets.

When a node $i$ receives a video packet, it will be enqued in the main buffer. Refer to fig 3. Eventually this video packet will reach the head of the main buffer, and it will be sent to the next node $(i+1)$. At this time, a copy of the transmitted video packet is kept in the retransmission buffer of node $i$.

If the packet sent from node $i$ to node $(i+1)$ gets dropped at node $(i+1)$ due to congestion, then, node $(i+1)$ will create a Retransmit Request Message Packet (RRMP), then copy the flow id and sequence number of the packet in it from the packet to be dropped. It will then send the RRMP to the upstream node $i$, only when the node $(i+1)$ has place in the buffer to accept the packet. This happens when the congestion gets over.

The RRMP from node $(i+1)$ is received by the upstream node $i$. This node, when receives an RRMP packet, it first finds the flow id and the sequence number. It then looks into its own retransmission buffer and checks if a copy of the required video packet (of the same flow id and sequence number) exists there. If it is present there, then that packet is retransmitted to the downstream node $(i+1)$. If the video packet of the same flow id and sequence number is not found in the retransmission buffer, then the received RRMP packet is ignored.

\section{RESULTS}

We conducted simulation study of the proposed N2N packet forwarding mechanism using a simulated network. The video source was a trace file of foreman.yuv video. The non-video data traffic used is Constant Bit Rate (CBR) traffic. 
The network considered for simulation study is shown in figure 4. This network uses three nodes. The first node (Node 1), receives data from a CBR data source S1. The data rate is $1600 \mathrm{kbps}$. The node 1 also receives the video data from a trace file for foreman video (Peak rate=1.04 Mbps, Average rate $=214 \mathrm{Kbps}$, and 400 frames). The bandwidth of link L1 is kept higher than the bandwidth of CBR source. Similarly the bandwidth of link L2 is kept slightly higher than the peak bandwidth required by the video source. The link delay of L3 is kept 160 milliseconds while all other links have delay of $1 \mathrm{~ms}$. This is done so as to simulate the network conditions with large RTT from video destination D2 to video source S2. The link L8 is used with bandwidth just above the average bandwidth of the video source so that it creates a bottleneck and causes some packet drop. The buffers for node 1 and node 2 are kept 52000 bytes and the same for node 3 is kept 1000 bytes. This is done so as to cause the packet drops at node 3 and to study its effect. Thus it is possible to study the effect of our proposed $\mathrm{N} 2 \mathrm{~N}$ algorithm on packet loss as well as large RTT. The performance metrics we used are:
1. \% Packet loss
2. Frames lost
3. PSNR for each frame
4. Mean PSNR of all frames
5. End to end packet delay

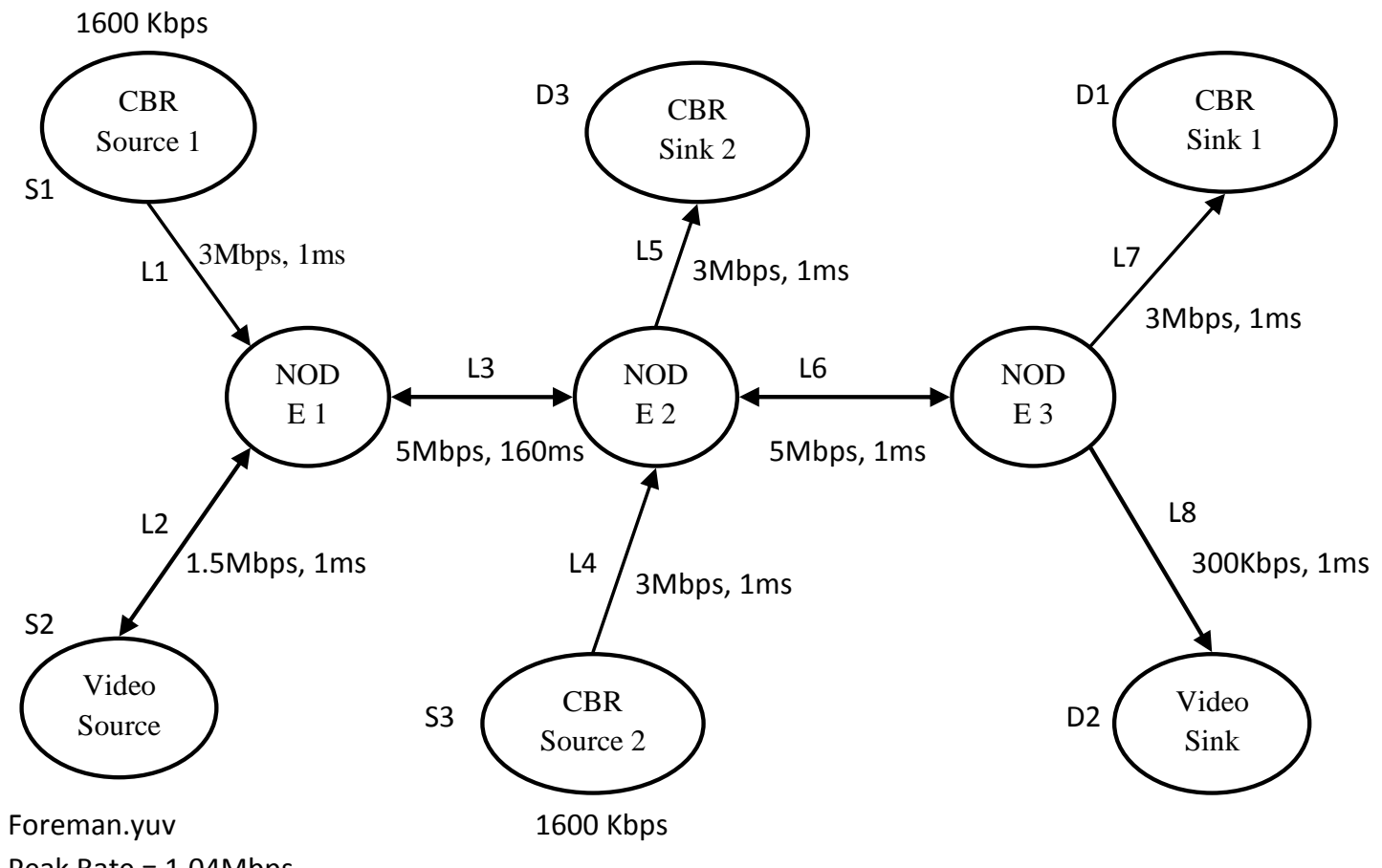

Peak Rate $=1.04 \mathrm{Mbps}$

Avg. Rate $=214 \mathrm{Kbps}$

Figure 4: Network for simulation study

We compared our algorithm with two more algorithms. The first being with no re-transmission and the second is TL-TCP (end-to end re-transmission with time out). To control the delay bound of TL-TCP we used adjustable retransmission buffer at the video source $\mathrm{S} 1$.

The results are shown in table 1 .

\begin{tabular}{|c|c|c|c|c|}
\hline & $\begin{array}{c}\text { \% ge } \\
\text { Video } \\
\text { Packet loss }\end{array}$ & $\begin{array}{c}\text { Retrans } \\
\text { Packets }\end{array}$ & $\begin{array}{c}\text { Frames } \\
\text { lost }\end{array}$ & PSNR \\
\hline $\begin{array}{c}\text { No } \\
\text { Retransmission }\end{array}$ & $2.71 \%$ & 0 & 24 & 30.45 \\
\hline $\begin{array}{c}\text { TL-TCP } \\
\text { Retransmission }\end{array}$ & $2.83 \%$ & 1 & 25 & 32.29 \\
\hline $\begin{array}{c}\text { N2N } \\
\text { Retransmission }\end{array}$ & $0.47 \%$ & 25 & 4 & 33.18 \\
\hline
\end{tabular}

Due to bottleneck link L8, there will be packet loss for video traffic at Node 3 only. So if a video packet is lost at Node 3, it will send a retransmit request to source $\mathrm{S} 2$ under TL-TCP algorithm. Under the proposed $\mathrm{N} 2 \mathrm{~N}$ algorithm however, node 3 will send retransmission requires to $\mathrm{N} 2$ and not to $\mathrm{S} 2$. This reduces the packet delay for the retransmitted packet.

It can be observed from the above table that, the \%ge packet loss is higher for no retransmission. For TL-TCP retransmission, the packet loss is nearly the same. This was because the retransmission buffer at the video source was kept smaller. For both these algorithms, the frame loss was found to be higher.

The Node-to Node retransmission algorithm was found to perform better since the packet loss was reduced to $0.47 \%$. The number of re-transmitted packets was 25 . This retransmission is from node 2 to node 3 and not from source $\mathrm{S} 2$ to Node 3. The re-transmission buffer for N2N algorithm was kept to be of 5 video packets.

Table 1. Performance Comparison 
The PSNR performance of the three algorithms is shown in figure 5, 6 and 7. Figure 5 shows the PSNR for no retransmission, Figure 6 shows the PSNR performance for TL-TCP re-transmission and Figure 7 shows the PSNR performance of the proposed $\mathrm{N} 2 \mathrm{~N}$ re-transmission based packet forwarding algorithm.

It was observed that, the PSNR of first two algorithms is almost the same whereas the $\mathrm{N} 2 \mathrm{~N}$ re-transmission algorithm indicates significant improvement in PSNR. This is due to the fact that, each node in the path of the video traffic uses a retransmission buffer and can retransmit the packet dropped downstream from there itself.

The mean PSNR was also found to have increased in the proposed algorithm. (Refer table 1)

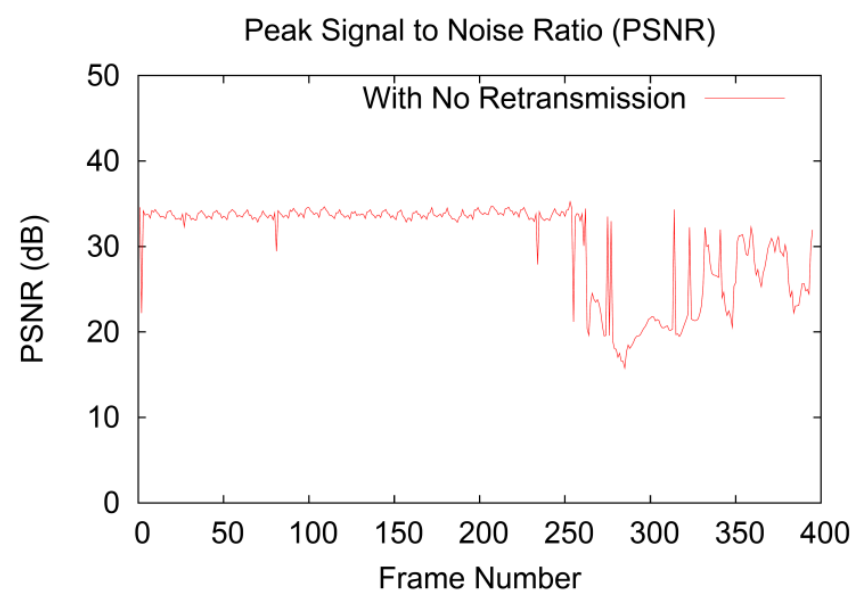

Figure 5: PSNR with no retransmission

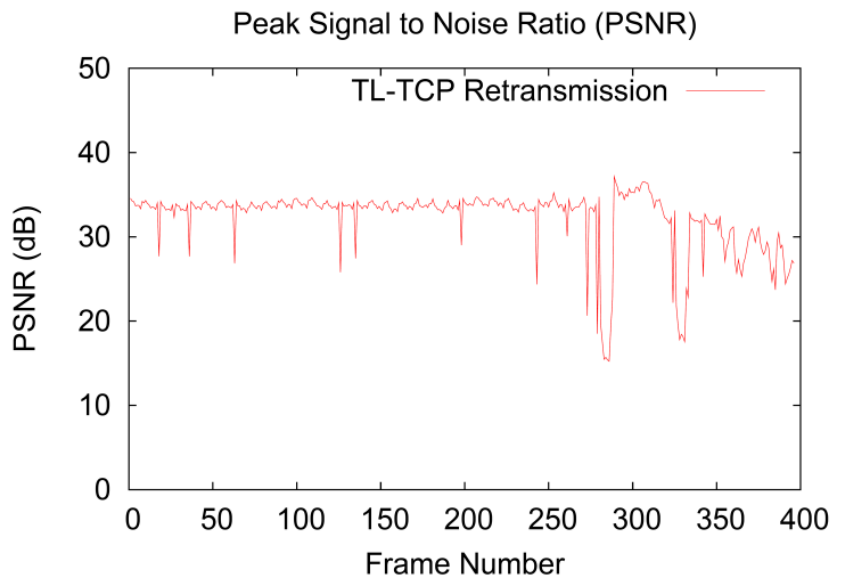

Figure 6: PSNR with TL-TCP retransmission
To test the jitter performance, we increased the retransmission buffer of TL-TCP algorithm at video source to 50 video packets. Due to this, a lot of re-transmissions of TL-TCP were successful, thus reducing the packet loss for TL-TCP algorithm. However, it caused the round trip delay to be large for re-transmitted video packets, thus increasing the jitter.

The figures 8, 9, 10 show that the end- to end video packet delay performance for no retransmission, TL-TCP retransmission and $\mathrm{N} 2 \mathrm{~N}$ re-transmission respectively.

The delay performance is shown in figures 8,9 , and 10 indicates that the TL-TCP has an advantage of low packet loss but suffers from large round trip delay for re-transmitted packets. This may cause the re-transmitted video packets to arrive late and hence the receiver may not be able to play them. Thus, it shows that, the proposed N2N re-transmission based packet forwarding algorithm is better as compared with TL-TCP algorithm in packet loss as well as delay performance.

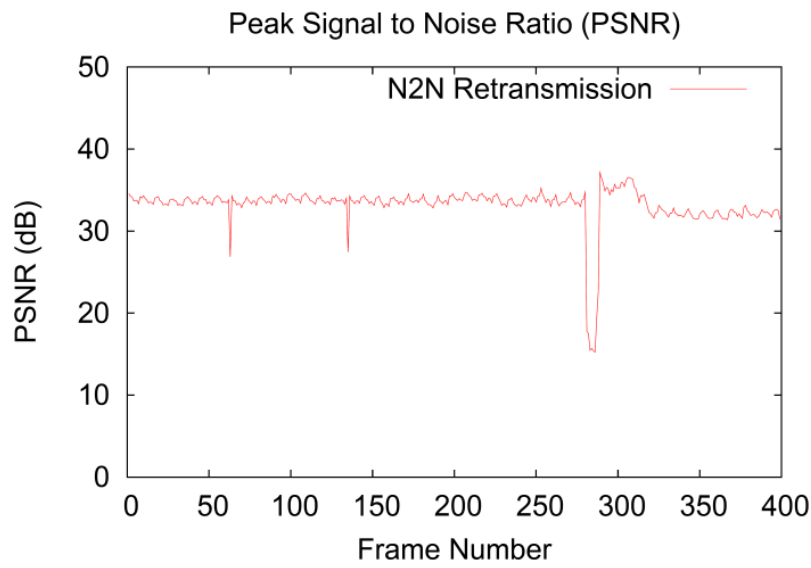

Figure 7: PSNR with Proposed N2N retransmission

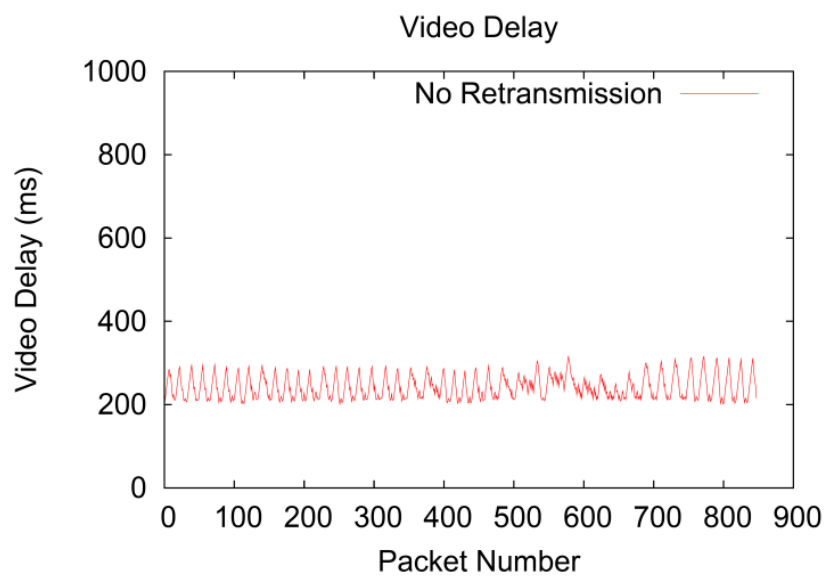

Figure 8: End- to end video packet delay for No retransmission 


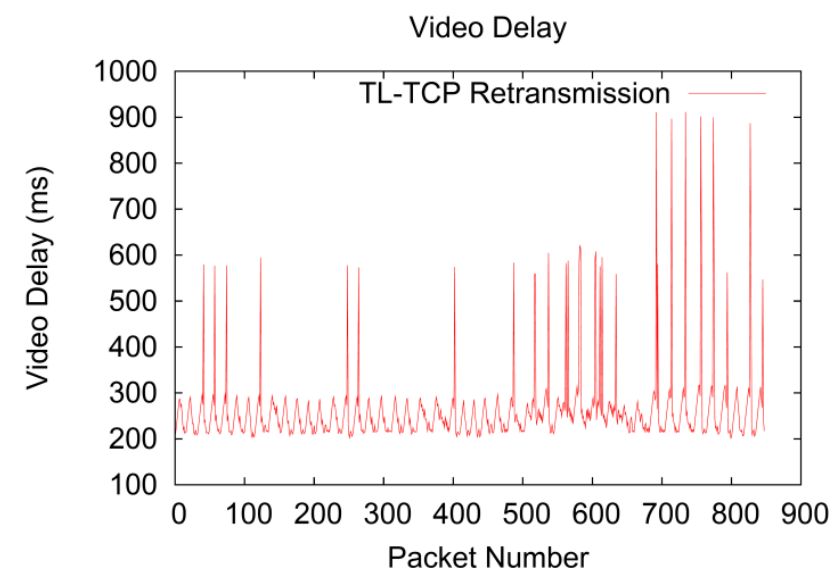

Figure 9: End- to end video packet delay for TL-TCP re-transmission Video Delay

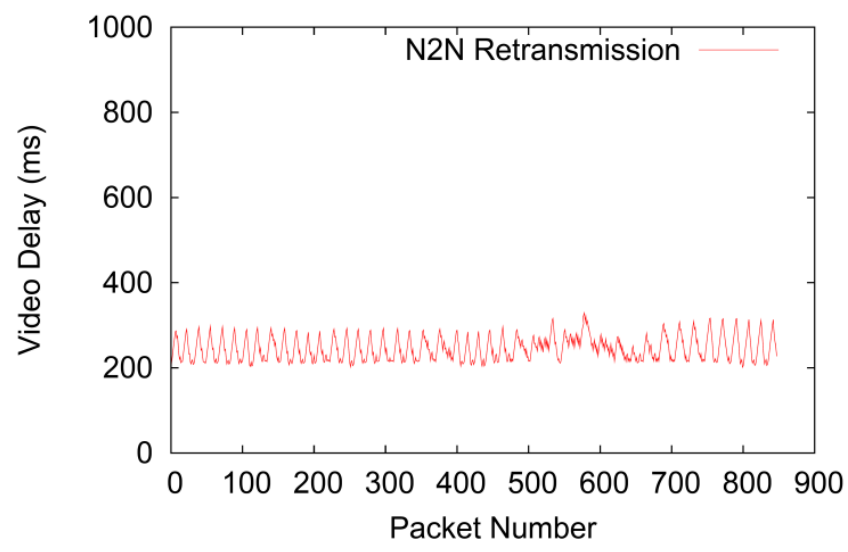

Figure 10: End- to end video packet delay for $\mathbf{N} 2 \mathrm{~N}$ re-transmission

\section{CONCLUSION}

In this paper, we presented a new Node to Node retransmission based packet forwarding mechanism for video traffic in IP networks. The proposed algorithm is shown to perform better in terms of \%ge packet loss and packet delay for video traffic as compared with TL-TCP re-transmission based algorithm.

Work is in progress to formulate a protocol to implement the proposed algorithm so that it can be realized in the present IP network.

\section{REFERENCES}

[1] Gall D. L. 1991, MPEG: A Video Compression Standard for Multimedia Applications, Communications of ACM, Volume 34, No. 4 Pg. 46-58

[2] Iain E. G. Richardson, 2003, H264 and MPEG-4 video compression, Video Coding for Next Generation Multimedia, John Wiley and Sons ISBN:0-470-84837-5

[3] Yuan-Chi Chang, Thom Carney et.al. 1998, Effects of Temporal Jitter on Video Quality: Assessment Using Psychophysical Methods, Proceedings of the SPIE: Human Vision and Image Processing.

[4] Amy R. Reibman and David Poole, AT\&T Labs, Characterizing packet-loss impairments in compressed video.

[5] Biswaroop Mukherjee, 2000, Time-lined TCP: a Transport Protocol for Delivery of Streaming Media over the Internet, Thesis at University of Waterloo. 